\title{
Bajos niveles de colesterol HDL es un predictor de mortalidad y de fibrilación auricular postoperatoria posterior a la cirugía de revascularización miocárdica
}

\author{
César Vargas ${ }^{1}$, Diego Vargas ${ }^{2}$, Víctor Neira ${ }^{3}$, Rodrigo Binder ${ }^{1}$, Christian Backhouse ${ }^{1}$, Braulio \\ Bobadilla ${ }^{1}$, Andrés Díaz ${ }^{4}$, Rodrigo Miranda ${ }^{3}$, Benjamín Stockins ${ }^{3}$, Fernando Lanas ${ }^{3}$
}

\author{
Unidad de Paciente Crítico, Hospital Hernán Henríquez Aravena \\ Facultad de Medicina, Universidad de la Frontera \\ 1. Residente de Cardiología, Universidad de la Frontera \\ 2. Interno de Medicina, Escuela de Medicina, Universidad Austral de Chile \\ 3. Cardiólogo, Departamento Cardiovascular, Universidad de la Frontera \\ 4. Cardiocirujano, Departamento Cardiovascular, Universidad de la Frontera
}

Introducción: Se ha demostrado que bajos niveles de colesterol HDL (C-HDL) se asocian a una mayor incidencia de fibrilación auricular y de mortalidad global y cardiovascular. En un estudio observacional previo en nuestro centro, encontramos que un bajo nivel de C-HDL se asoció a mayor riesgo de fibrilación auricular postoperatoria (FAPO) en pacientes sometidos a cirugía de revascularización miocárdica (CRM).

Objetivo: Evaluar si el bajo nivel de C-HDL se asocia a mayor incidencia de FAPO y mortalidad en un seguimiento a un año en un estudio controlado.

Método: Se realizó un estudio observacional prospectivo que incluyó a 100 pacientes consecutivos sometidos a CRM por enfermedad coronaria estable sin antecedentes de FA y que ingresaron a la UCI Cardioquirúrgica en ritmo sinusal. Se definió FAPO como FA con duración mayor a 5 minutos o 5 episodios de FA mayores a 30 segundos de duración en los primeros 5 días post operatorios. Se consideró bajo nivel de C-HDL a un valor $<30 \mathrm{mg} / \mathrm{dL}$. Los pacientes se siguieron por un año. Se utilizó un análisis univariado y multivariado para identificar factores predisponentes de FAPO y mortalidad.

Resultados: 31 pacientes presentaron FAPO. El análisis multivariado mostró un incremento de FAPO con C-HDL $<30 \mathrm{mg} / \mathrm{dL}$ (OR 5.01, IC95\% 1.3-18.8, $\mathrm{p}=0,017$ ) y con albúmina $<3,5 \mathrm{gr} / \mathrm{dL}$ (OR 6.42, IC95\% 1.58-26.0, $\mathrm{p}=0,009)$. En un seguimiento de $14.1 \pm 1.7$ meses. La mortalidad global fue $6 \%$ y un C-HDL $<30 \mathrm{mg} / \mathrm{dL}$ resultó ser un predictor independiente (HR 11.1, IC95\% 1.1-38.4, $\mathrm{p}=0,039$ ).

Conclusión: En nuestra serie un C-HDL menor a $30 \mathrm{mg} / \mathrm{dL}$ es un predictor independiente de FAPO y mortalidad posterior a la CRM. 


\section{Low HDL level is a predictor of Post Operative Atrial fibrillation and mortality after coronary artery bypass surgery}

Background: Low C-HDL level has been associated to an increased incidence of atrial fibrillation (AF) and cardiovascular mortality. Previously, we have observed that low C-HDL had the same type of association with post operative AF (POAF) and mortality following surgery for coronary artery disease.

Aim: to evaluate whether a low C-HDL level is a predictor of POAF and mortality following revascularization surgery in a controlled study.

Method: A prospective observational study included 100 consecutive patients undergoing revascularization surgery for stable coronary artery disease (CAD) in sinus rhythm and no prior AF. POAF was defined as AF sustained for more than 5 min or the occurrence of 5 or more episodes of AF extending for more than 5 seconds during the first 5 post operative days. A value $<30 \mathrm{mg} / \mathrm{dl}$ was considered low C-HDL.
Patients were followed for one year. Uni and multivariate analysis were used to identify predictors of POAF and mortality.

Results: 31 patients developed POAF. A significant $(\mathrm{p}=0.017)$ OR of $5.01(95 \%$ CI $1.3-18.8)$ between low C-HDL and POAF was shown. A similar association linked low serum albumin level to POAF (OR 6.4, C.I. 1.6 - 26). After $14.1 \pm 1.7$ months of follow-up global mortality was $6 \%$. Low C-HDL turned out to be a significant predictor of mortality (H.R. 11.1, C.I. $1.1-38.4, \mathrm{p}=0.04)$.

Conclusion: Low C-HDL is an independent predictor of POAF and mortality after coronary artery revascularization surgery.

Keywords, coronary artery, bypass surgery, atrial fibrillation, HDL cholesterol, mortality. 
Introducción: La cirugía de revascularización miocárdica (CRM) es la principal estrategia terapéutica para reducir la mortalidad en la enfermedad coronaria. En nuestro centro se realizan alrededor de 225 procedimientos anuales de este tipo.

La fibrilación auricular postoperatoria (FAPO) es una complicación médica frecuente que influye en el pronóstico a corto y largo plazo. Tiene una incidencia reportada entre 20 y $50 \%$ dependiendo de la definición y método de detección3. Se han descrito varios factores independientes para la aparición de FAPO, siendo la edad uno de los más recurrentes en la literatura. También se han mencionado la disminución de la fracción de eyección del ventrículo izquierdo (FEVI), sexo femenino, diabetes mellitus, enfermedad renal crónica, enfermedad pulmonar obstructiva y una enfermedad severa de la coronaria derecha $(\mathrm{ESCD})^{8}$. Más recientemente se ha reportado que la obesidad y el síndrome metabólico son factores independientes de $\mathrm{FAPO}^{7}$, 12,13. También se ha reportado que el uso de drogas para la prevención de FA como el uso de betabloqueadores y amiodarona puede reducir la mortalidad a largo plazo en este grupo de pacientes $7,18,19,20$.

Los trabajos a largo plazo han mostrado que un importante factor predisponente de mortalidad siguiendo a la cirugía coronaria es la FAPO. La principal causa de mortalidad a largo plazo asociada a FAPO es la etiología cardíaca (50$60 \%$, principalmente por insuficiencia cardíaca), seguida por el accidente cerebro vascular, cáncer, y menos frecuentemente, la infección ${ }^{3,4,5}$.

Estudios prospectivos han mostrado que el Colesterol HDL (C-HDL) disminuye con la edad producto de cambios hormonales, procesos inflamatorios y diabetes mellitus ${ }^{6}$. Un estudio de seguimiento a 15 años mostró que un bajo nivel de C-HDL fue el principal factor metabólico de mortalidad global ${ }^{2}$.

En un estudio realizado en nuestro centro encontramos que un bajo nivel de C-HDL fue un factor independiente de FAPO y que el uso de betabloqueadores cardioselectivos (BBCS) resulto un factor protector ${ }^{10}$.

El objetivo de este trabajo fue conocer si bajos niveles de C-HDL pueden ser factores predisponentes e independientes de FAPO y mortalidad global.

\section{Pacientes y métodos}

Se realizó un estudio prospectivo observacional en el periodo entre Abril y Septiembre de 2013. Los criterios de inclusión fueron: pacientes consecutivos sometidos a cirugía de revascularización miocárdica por enfermedad coronaria estable, que no presentaban antecedentes clínicos de fibrilación auricular, estuvieran en ritmo sinusal y que habían permanecido al menos 5 días de post operatorio en la Unidad de Paciente Crítico Cardiovascular (UPC-CV). Se consideraron elegibles para el estudio a 100 de 113 pacientes que se operaron de CRM durante ese período.

Se consideró enfermedad coronaria estable aquella cursando con angina estable y, en el caso de pacientes que presentaron síndrome coronario agudo, aquellos que evolucionaron al menos 21 días sin complicaciones mayores. Se definió FAPO como FA que tuviera una duración mayor a 5 minutos o 5 episodios de FA con duración mayor a 30 segundos. El registro se llevó a cabo mediante monitoreo de telemetría Sistema Dräger Infinity ${ }^{\circledR}$ CentralStation evaluando diariamente los eventos arrítmicos y su duración. La observación se realizó durante los primeros cinco días del postoperatorio.

Los factores considerados para el análisis fueron predictores conocidos para FAPO encontrados en la literatura. Para su análisis estadístico se definieron de la siguiente forma: sexo masculino y femenino, edad mayor o igual a 70 años, Colesterol LDL (C-LDL) mayor o igual a $100 \mathrm{mg} /$ dL, C-HDL menor a 30mg/dL, albúmina menor a 3,5gr/ dL, HbA1c mayor o igual a $6,5 \%$, FEVI menor a $50 \%$ por ecocardiografía o ventriculografía y ESCD como lesiones mayores al $70 \%$ en su tercio proximal o medio.

Además, en forma retrospectiva se analizó el efecto de los distintos betabloqueadores administrados. Se definió como uso precoz cuando los betabloqueadores eran iniciados durante las primeras 24 horas desde la llegada del paciente a la Unidad de Cuidados Críticos Cardioquirúrgica y administrados mientras este se mantuviera en ritmo sinusal. Para el análisis se dividieron en cardioselectivos (BBCS) o no.

El análisis estadístico se realizó con el programa SPSS versión 20.0. Para la determinación de significancia estadística de los factores predisponentes de FAPO y el efecto protector de los betabloqueadores se consideró estadísticamente significativo todo valor de $\mathrm{p}$ menor a 0,05 por método de chi cuadrado o test exacto de Fisher bilateral, según correspondiera a la variable. Para el análisis multivariado se utilizó regresión logística binaria para determinar Odd Ratio con intervalo de confianza del 95\% (IC95\%) y significancia estadística $p=<0,05$. Se incluyeron variables que en el análisis univariado resultaron significativas o presentaran un valor de $\mathrm{p}<0,5$.

Para el estudio de sobrevida se realizó un seguimiento de los pacientes por vía telefónica y en caso de fallecimiento se realizó una evaluación de la historia clínica para determinar la causa de muerte. La sobrevida se analizó con 
el método de Kaplan-Meier y los grupos se compararon mediante log rank test. Para determinar los factores independientes se realizó un análisis univariado y multivariado por regresión de Cox para determinar Hazard Ratio con intervalo de confianza del 95\%. Se consideró estadísticamente significativo un valor de $\mathrm{p}<0,05$. Se incorporó en el análisis multivariado las variables significativas y aquellas con un valor de $\mathrm{p}<0,4$. También se incorporó la edad por ser un predictor de mortalidad.

Se contó con autorización del comité de ética del nuestro recinto asistencial para la utilización de los datos.

\section{Resultados}

Se incluyeron 100 pacientes consecutivos. Su edad promedio fue 62,8 $\pm 9,1$ años, $76 \%$ fueron hombres, $63 \%$ tenía diabetes mellitus tipo 2 (DM2), 68\% HTA y $72 \%$ el antecedente de síndrome coronario agudo en los dos meses previo a la CRM. El C-HDL promedio fue $36,3 \pm$ $8,9 \mathrm{mg} / \mathrm{dL}$, levemente menor a lo reportado por la literatura en este grupo de pacientes; $\mathrm{C}$-LDL promedio fue $94,2 \pm 32,6 \mathrm{mg} / \mathrm{dL}$, la HbA1c $6,6 \pm 1,4 \%$ y la albúmina $3,69 \pm 0,35 \mathrm{~g} / \mathrm{dL}$.

Se implantó un promedio de 2,2 $\pm 0,5$ puentes aortocoronarios, destacando la anastomosis de arteria mamaria interna izquierda a la coronaria descendente anterior en el $97 \%$ de los pacientes y puentes aorto coronario con vena safena en el $78 \% .88$ pacientes se operaron con circulación extracorpórea con un tiempo promedio de 72,3 $\pm 25,2 \mathrm{~min}$. 31\% de los pacientes desarrolló FAPO y no hubo relación estadística con los factores operatorios mencionados.

Se asociaron significativamente a FAPO el C-HDL $<30 \mathrm{mg} / \mathrm{dL}$, una albúmina $<3,5 \mathrm{~g} / \mathrm{dL}$ y el uso de BBCS (Tabla 1). El análisis multivariado permitió estimar que un valor de C-HDL $<30 \mathrm{mg} / \mathrm{dL}$ aumentó en aproximadamente 5 veces el riesgo de FAPO y tener una albúmina $<3,5 \mathrm{~g} /$ dL lo aumentó en 6 veces. Se administró BB a $100 \%$ de los pacientes durante las primeras 24 horas postoperatorias (Bisoprolol 36\%, Propanolol 30\%, Carvedilol 16\%, Atenolol $16 \%$ y Nevibolol 2\%). El análisis multivariado mostró que el uso precoz de BBCS fue un factor protector de FAPO (OR 0.12). La edad $\geq 70$ años y HbA1c $\geq 6,5 \%$ también se asociaron significativamente a la aparición de FAPO ( $\mathrm{p}=0.05)$ (Tabla 2), pero no alcanzaron significancia estadística.

En está serie, 96\% de los paciente fueron dados de alta utilizando algún betabloqueador, $98 \%$ utilizando estatinas (93\% atorvastatina) y todos utilizando aspirina. Fueron seguidos por $14.1 \pm 1.7$ meses. Fallecieron 6 pacientes en total, 3 de ellos por causa cardíaca, 2 por accidente cerebro
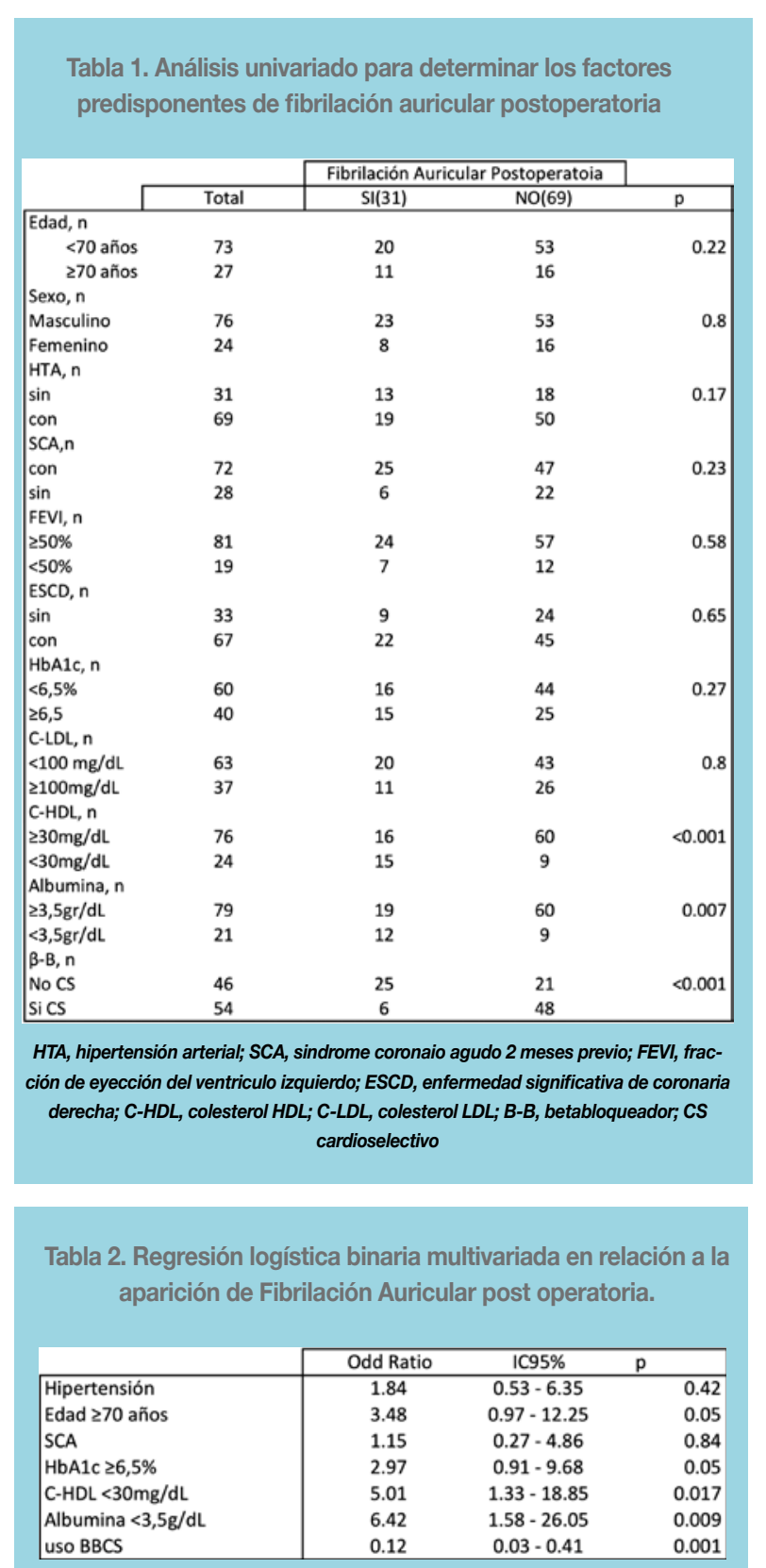

SCA, síndrome coronario agudo 2 meses previo; BBCS, betabloqueador cardioselectivo; C-HDL, colesterol HDL.

vascular isquémico y uno por shock séptico alejado de la CRM. 4 de los 6 fallecidos habían desarrollado FAPO. En la Figura 1 (Kaplan Meier) se observa que la sobrevida a 1 año fue significativamente mayor en los pacientes con C-HDL $\geq 30 \mathrm{mg} / \mathrm{dl}$ que en aquellos con un valor menor (98.7 vs 70.2, respectivamente, $\mathrm{p}<0.001$ ). Por otra parte, la FAPO no se asoció significaticamente a menor sobrevida p 0,057. En el análisis de regresión univariada de Cox se mostró que solo la variable C-HDL $<30 \mathrm{mg} / \mathrm{dl}$ se relacionó significativamente con menor sobrevida. 
Figura 1. Curva de Kaplan-Meier que muestra la supervivencia a 1 año, según el rango de Colesterol HDL.

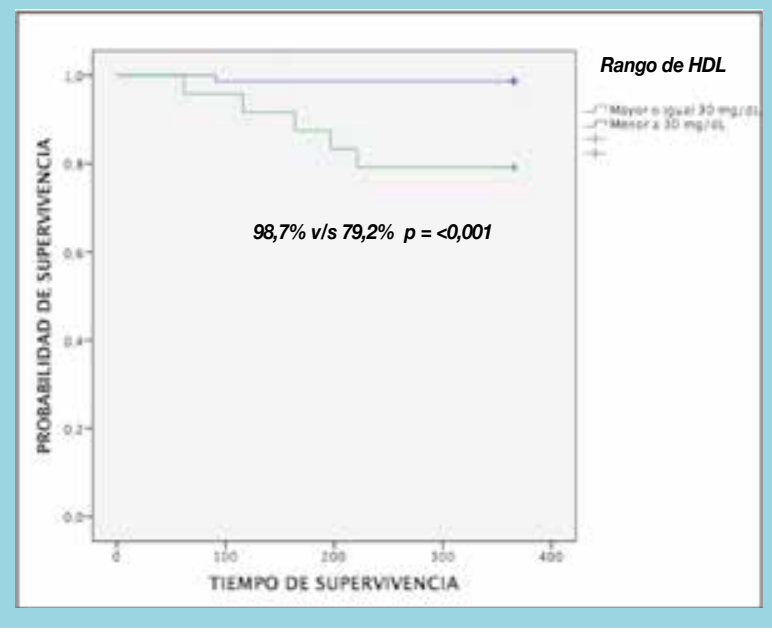

Finalmente, el análisis multivariado por regresión de Cox (Tabla 4) sugiere que un C-HDL $<30 \mathrm{mg} / \mathrm{dL}$ aumenta aproximadamente 11 veces el riesgo de fallecer a un año después de una CRM y que un $\mathrm{C}$-LDL $\geq 100 \mathrm{mg} / \mathrm{dL}$ lo hace en 5 veces. Ambas variables fueron predictores independientes de mortalidad global.

\section{Discusión}

Con los datos obtenidos en nuestro estudio podemos concluir que presentar un Colesterol HDL menor a 30mg/dL es un predictor independiente de FAPO y de mortalidad global a un año. Estos resultados son concordantes con varios estudios, tanto epidemiológicos, como realizados en pacientes sometidos a CRM. Así, en el año 2000 Foody et $\mathrm{al}^{2}$, con un seguimiento de 15 años muestran un signi-

Tabla 3. Análisis univariado por regresión de Cox para identificar factores predisponentes de mortalidad global posterior a la Cirugía de Revascularización Miocárdica

\begin{tabular}{|l|ccr|}
\cline { 2 - 4 } \multicolumn{1}{c|}{} & HR & IC $95 \%$ & $\mathrm{p}$ \\
\hline Edad $\geq 70$ años & 1.38 & $0.25-7.57$ & 0.70 \\
FEVI $<50 \%$ & 2.13 & $0.39-11.66$ & 0.38 \\
Hipertensión & 0.41 & $0.05-3.58$ & 0.42 \\
Albumina $<3,5 \mathrm{~g} / \mathrm{dL}$ & 1.87 & $0.34-10.26$ & 0.46 \\
C-LDL $\geq 100 \mathrm{mg} / \mathrm{dL}$ & 3.52 & $0.64-19.21$ & 0.14 \\
C-HDL $<30 \mathrm{mg} / \mathrm{dL}$ & 11.17 & $1.30-42.35$ & 0.028 \\
FAPO & 4.50 & $0.82-24.59$ & 0.082 \\
No uso de BBCS & 6.04 & $0.70-51.71$ & 0.10 \\
\hline
\end{tabular}

FEVI, fracción de eyección del ventriculo izquierdo; C-LDL, colesterol LDL; C-HDL, colesterol HDL; FAPO, fibrilación auricular postoperatoria; BBCS, betabloqueadores cardioselectivos; HR, hazard ratio; IC9\%, intervalo de confianza del $95 \%$.
Tabla 4. Análisis multivariado por regresión de Cox para identificar factores predisponentes de mortalidad global posterior a la Cirugía de Revascularización Miocárdica

\begin{tabular}{|l|ccr|}
\cline { 2 - 4 } \multicolumn{1}{c|}{} & HR & IC 95\% & p \\
\hline Edad $\geq 70$ años & 1.53 & $0.24-9.62$ & 0.64 \\
FEVI $<50 \%$ & 1.47 & $0.22-9.58$ & 0.68 \\
Albumina $<3,5 \mathrm{~g} / \mathrm{dL}$ & 0.74 & $0.60-8.02$ & 0.80 \\
C-LDL $\geq 100 \mathrm{mg} / \mathrm{dL}$ & 5.66 & $1.01-31.86$ & 0.049 \\
C-HDL $<30 \mathrm{mg} / \mathrm{dL}$ & 11.07 & $1.13-38.44$ & 0.039 \\
FAPO & 1.52 & $0.20-11.51$ & 0.68 \\
No uso de BBCS & 3.33 & $0.27-41.45$ & 0.34 \\
\hline
\end{tabular}

FEVI, fracción de eyección del ventrículo izquierdo; C-LDL, colesterol LDL; C-HDL, colesterol HDL; FAPO, fibrilación auricular postoperatoria; BBCS, betabloqueadores cardioselectivos; HR, hazard ratio; IC9\%, intervalo de confianza del $95 \%$.

ficativo aumento en mortalidad alejada en pacientes con $\mathrm{C}-\mathrm{HDL} \leq 35 \mathrm{mg} / \mathrm{dL}(74 \% \mathrm{v} / \mathrm{s} 57 \%)$, concluyendo que es el factor metabólico más importante como predictor de mortalidad a largo plazo $^{2}$. Este hecho es concordante con el efecto de activación plaquetaria y pro trombótico de una relación LDL/HDL elevada ${ }^{21,22}$. Si bien la relación de niveles bajos de C-HCL con FAPO está establecida, la magnitud de su influencia ha sido variable en diferentes estudios, lo que puede relacionarse con los distintos criterios de definición de FAPO.

Respecto de la relación entre FAPO y sobrevida postoperatoria alejada, Villareal et al encontraron una sobrevida a 5 años de $74 \%$ en pacientes que habían desarrollado FAPO comparada con $87 \%$ en los que no había presentado dicha arritmia $^{3}$. En el presente estudio, en cambio, dicha relación no se hizo evidente, lo que podría atribuirse al escaso número de pacientes fallecidos.

En este estudio encontramos que presentar una albúmina $<3,5 \mathrm{~g} / \mathrm{dL}$ fue un predictor independiente de FAPO, pero no de mortalidad, hecho que no tiene una explicación. Es conocida la relación entre aumento de la actividad adrenérgica y la aparición de FAPO ${ }^{9.14}$, pero no tenemos manera de relacionar dicho aumento con la hipoalbuminemia.

El presente estudio mostró que los BBCS administrados durante las primeras 24 horas a sujetos en ritmo sinusal fue una estrategia beneficiosa para reducir la incidencia de FAPO. El bisoprolol fue el BBCS más utilizado en tanto que el BB no cardioselectivo más usado fue el propranolol. Por lo tanto, nuestros hallazgos concuerdan con los de otros autores que reportan incidencias de FAPO entre 10 y $12 \%$ con bisoprolol comparado con 30 a $40 \%$ en sujetos que recibieron propranolol ${ }^{18-20}$.

La limitación más importante del estudio es el relativamente bajo número de sujetos incluidos y, en especial, el menor poder estadístico para detectar diferencias signifi- 
cativas de mortalidad con solo 6 pacientes fallecidos. En nuestra serie los pacientes presentaron menores niveles de C-HDL que lo reportado en otras series, lo que sumado al bajo número de pacientes puede explicar el valor alto del HR y lo amplio de su intervalo de confianza.

En conclusión, el presente estudio refuerza hallazgos pre- vios que relacionan bajos niveles de C-HDL con el desarrollo de FAPO y con menor sobrevida alejada en pacientes sometidos a revascularización miocárdica quirúrgica. Dada la ausencia de tratamientos farmacológicos para elevar el C-HDL no es posible por ahora probar el efecto causal de esta relación.

\section{Referencias:}

1. YUSUF S, ZUCKER D, PEDUZZI P, FISHER LD, TAKARO T, KENNEDY JW, et al. Effect of coronary artery bypass graft surgery on survival: overview of 10-year results from randomised trials by the Coronary Artery Bypass Graft Surgery Trialists Collaboration. Lancet. 1994; 344: 563-70.

2. FOODY JM, FERDINAND FD, PEARCE GL, LYTLE BW, COSGROVE DM, SPRECHER DL. HDL cholesterol level predicts survival in men after coronary artery bypass graft surgery: 20-year experience from The Cleveland Clinic Foundation. Circulation. 2000;102: III-90-III-94.

3. VILLAREAL RP, HARIHARAN R, LIU BC, KAR B, LEE VV, ELAYDA M, et al. Postoperative atrial fibrillation and mortality after coronary artery bypass surgery. J Am Coll Cardiol. 2004; 43: 742-748.

4. BRADSHAW PJ, JAMROZIK K, LE M, GILFILLAN I, THOMPSON PL. Mortality and recurrent cardiac events after coronary artery bypass graft: Long term outcomes in a population study. 2002; 88: 488-494.

5. HERLITZ J, BRANDRUP-WOGNSEN G, CAIDAHL K, HAGLID-EVANDER M, HARTFORD M, KARLSON B, et al. Cause of death during 13 years after coronary artery bypass grafting with emphasis on cardiac death. Scand Cardiovasc J.
2004; 38: 283-286.

6. WALTER M. Interrelationships among HDL metabolism, aging, and atherosclerosis. Arterioscler Thromb Vasc Biol. 2009; 29: 1244:1250.

7. ECHAHIDI N, PIBAROT P, O'HARA G, MATHIEU P. Mechanism, prevention, and treatment of atrial fibrillation after cardiac surgery. J Am Coll Cardiol. 2008; 51: 793-801.

8. MENDES L, CONNELLY G, MCKENNEY P, PODRIS P, CUPPLES A, SHEMIN R, et al. Rigth coronary artery stenosis: an independent predictor of atrial fibrillation after coronary bypass surgery. J Am Coll Cardiol. 1995; 25: 198-202.

9. MONTAIGNE D, MARECHAL X, LEFEBVRE P, MODINE T, FAYAD G, DEHONDT H, et al. Mitochondrial dysfunction as an arrhythmogenic substrate: a translational proof-of-concept study in patients with metabolic syndrome developping post-operative atrial fibrillation. J Am Coll Cardiol. 2013; 62:1466-73.

10. VARGAS C, VARGAS D, PEÑA M, ASSEF V, DÍAZ A, MIRANDA R, et al. Niveles bajos de colesterol HDL y albumina son factores de riesgo independientes de fibrilación auricular posterior a cirugía de revascularización miocárdica. Rev Chil Cardiol; Libro de resúmenes Congreso 2013: 60-61. 
11. OKIN P, HILLE D, WACHTELL K, KJELDSEN S, DAHLÖF B, DEVEREUX R. Low in-treatment HDL colesterol strongly predicts incidente atrial fibrillation: The LIFE study. Circulation. 2012; 126: A9371.

12. ZACHARIAS A, SCHAWNN T, RIORDAN C, DURHAM S, SHAH A, HABIB R. Obesity and risk of new-onset atrial fibrillation after cardiac surgery. Circulation. 2005; 112: 32473255 .

13. ECHAHIDI N, MOHTY D, PIBAROT P, DESPRES J, O'HARA T, CHAMPAGNE J, et al. Obesity and metabolic syndrome are independent risk factors for atrial fibrillation after coronary artery bypass surgery. Circulation. 2007;116:1213-1219.

14. GAUDINO M, ANDREOTTI F, ZAMPARELLI R, DI CASTELNOUVO A, NASSO G, BURZOTTA F, et al. The -174G/C interleukin-6 polymorphism influences postoperative interleukin-6 levels and postoperative atrial fibrillation. Is atrial an inflamatory complication?. Circulation 2003; 108: II195-199.

17. KONIARI I, APOSTOLAKIS E, ROGKADUKOU C, BAIKOOUSSIS, DOUGENIS D. Pharmacologic prophylaxis for atrial fibrillation following cardiac surgery: a systemic review. J Cardiothorac Surg. 2010; 5: 121-131.
18. COLEMAN C, PERKERSON K, GILLESPIE E, KLUGER J, GALLAGHER R, HOROWITZ S, et al. Impact of prophylactic postoperative beta-blockade on post-cardiothoracic surgery lenght of stay and atrial fibrillation. Ann Pharmacother. 2004;38:2012-2016.

19. SLEILATY G, MADI-JEBARA S, YAZIGI A, HASSAD F, HAYECK G, EL RASSI I, et al. Postoperative oral amiodarone versus oral bisoprolol as prophylaxis against atrial fibrillation after coronary bypass graft surgery: a prospective randomized trial. Int J Cardiol. 2009; 137: 116-122.

20. MARAZZI G, IELLAMO F, VOLTERRANI M, CAMINITI G, MADONNA M, ARISI G, et al. Comparison of effectiveness of carvedilol versus bisoprolol for prevention of postdischarge atrial fibriallation after coronary artery bypass graffting patients with heart failure. Am J Cardiol. 2011; 107: 215-9.

21. NOFER JR1, BRODDE MF, KEHREL BE. High-density lipoproteins, platelets and the pathogenesis of atherosclerosis. Clin Exp Pharmacol Physiol. 2010; 37: 726-35.

22. TSIMIHODIMOS V, KARABINA SA, TAMBAKI AP, BAIRAKTARI E, MILTIADOUS G, GOUDEVENOS JA, et al. Altered distribution of platelet-activating factor- acetylhydrolase activity between LDL and HDL as a function of the severity of hypercholesterolemia. J Lipid Res 2002; 43: 256- 263. 